DOI: $10.4274 /$ jarem.galenos.2020.3304

J Acad Res Med 2020;10(3):227-31

\title{
Visual Evoked Potentials in Euthyroid Hashimoto's Thyroiditis
}

\author{
(1) Hakan Şilek1, (1) Rengin Bilgen1, (1) Bahar Erbaş2,3 \\ ${ }^{1}$ Yeditepe University Faculty of Medicine, Department of Neurology, İstanbul, Turkey \\ ${ }^{2}$ Demiroğlu Bilim University Faculty of Medicine, Department of Pharmacology, İstanbul, Turkey \\ ${ }^{3}$ istanbul Florence Nightingale Hospital, Clinic of Neurology, İstanbul, Turkey
}

Cite this article as: Şilek H, Bilgen R, Erbaş B. Visual Evoked Potentials in Euthyroid Hashimoto's Thyroiditis. J Acad Res Med 2020;10(3):227-31

\begin{abstract}
Objective: Hashimoto's thyroiditis (HT) is an autoimmune disease in which neurological involvement is not uncommon. This study aimed to explore the presence of visual evoked potential (VEP) changes as an indicator of subclinical central nervous system involvement in euthyroid HT patients without obvious neurologic findings.

Methods: Thirty HT patients with normal neurological examination and thirty healthy controls were included. VEPs were recorded by using patternreversing black and white checkerboard with monocular testing. P100, N75 and N135 (ms) peak latencies, and P100 amplitudes of right (R) and left (L) eyes in each group were compared.

Results: There was no significant difference between the groups for age. The mean of the P100 (R: 108.13 \pm 4.3, L: $108.2 \pm 4.4$ ms), N75 (R: $79.23 \pm 6.03$, $\mathrm{L}: 80.2 \pm 5.78 \mathrm{~ms}$ ) and N135 (R: $141.8 \pm 11.2, \mathrm{~L}: 142.4 \pm 10.2 \mathrm{~ms}$ ) latencies, and the P100 amplitude (R: $6.71 \pm 4.16, \mathrm{~L}: 6.6 \pm 3.9 \mu \mathrm{V})$ in the HT group were not

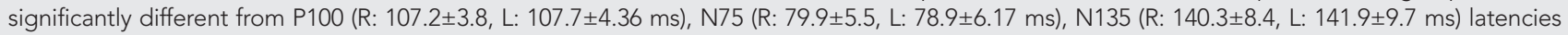
and P100 amplitude (R: $8.20 \pm 3.32, \mathrm{~L}: 6.9 \pm 2.9 \mu \mathrm{V}$ ) in the control group. Also, there was no significant correlation between P100 latencies and thyroid specific antibody levels in the HT group.

Conclusion: This result may be due to the fact that HT does not significantly affect the optic nerve and visual pathways, or that VEP is an inadequate technique to demonstrate possible involvement.

Keywords: Hashimoto's thyroiditis, visual evoked potentials, P100, N75, N135
\end{abstract}

\section{INTRODUCTION}

Hashimoto's thyroiditis (HT), also known as chronic lymphocytic thyroiditis or autoimmune thyroiditis may be associated with various neurological disorders, presenting as central and/or peripheral nervous system involvement (1-3). The detection of an increase in serum anti-thyroid peroxidase antibody (TPOAb) and anti-thyroglobulin antibody ( $\mathrm{TgAb}$ ) levels in this autoimmune disease is important for making the diagnosis. Hashimoto's encephalopathy (HE) is the most remarkable example of a central nervous system (CNS) involvement linked to HT (3). It has been shown that demyelinating lesions similar to multiple sclerosis (MS) and single-photon emission computed tomography abnormalities can be seen in HT $(4,5)$. Furthermore, brain function abnormalities such as cognitive and affective disturbances have also been reported in euthyroid HT patients with subtle neurological symptoms (6).

ORCID IDs of the authors: H.Ş. 0000-0002-6550-6200; R.B. 0000-0002-5138-971X; B.E. 0000-0002-6125-7761

Corresponding Author: Hakan Şilek,

E-mail: hakan.silek@yeditepe.edu.tr
Received Date: 28.03.2020 Accepted Date: 27.09.2020

(C) Copyright 2020 by University of Health Sciences Turkey, Gaziosmanpaşa Training and Research Hospital. Available on-line at www.jarem.org 
In many diseases of the CNS, particularly in demyelinating diseases, the visual system can be affected subclinically $(7,8)$. Visual evoked potential (VEP), a gross electrical signal recorded from the visual cortex in response to a changing visual stimulus on the check-board pattern (pattern onset/reversal VEP), is one of the methods used to demonstrate the overt or subtle dysfunctions of the visual system (9).

This study aimed to explore the presence of VEP changes as an indicator of subclinical CNS involvement in euthyroid HT patients without obvious neurologic findings.

\section{METHODS}

The participants of the patient group (HT group) were randomly selected (by simple randomization according to their protocol numbers) from the patients (over 18 years old) who were admitted to the neurology outpatient clinic from January to March 2019 with non-specific complaints and an HT diagnosis confirmed by an endocrinologist. All the patients had high TPOAb (>34 IU/ $\mathrm{mL})$ and $\mathrm{TgAb}(>115 \mathrm{IU} / \mathrm{mL})$ levels, reduced echogenicity of the thyroid parenchyma on thyroid ultrasonography, normal thyroid function tests, and no objective finding on the neurological examination. The control group consisted of healthy volunteers. The participants with ocular diseases that could affect the VEP test, such as glaucoma, were excluded from the study. None of the participants had visual complaints.

All participants underwent neurologic examination, and visual acuity was measured using a Snellen chart. Thyroid stimulating hormone (TSH), free thyroxine (fT4), TPOAb, and TgAb levels in the last three months were obtained retrospectively from the patient records.

VEPs were performed by the same technician, in the neurophysiology laboratory, using the 10/20 system monocular reverse pattern VEP method, with Keypoint G4 (Natus Medical Incorporated Alpine Biomed Apps Skovlund, Denmark). The VEP study protocol was based on the recommendations of the International Society for Clinical Electrophysiology of Vision on performing VEPs (10). The active electrode $(\mathrm{Oz})$ was placed in the midoccipital line, $5 \mathrm{~cm}$ superior to the inion. The reference VEP has been used in clinical neurology, for the electrode $(F z)$ was in the mid frontal line and the grounding electrode was at the vertex $(\mathrm{C} z)$. Visual stimulation was provided by a pattern generator monitor with a mean luminance of $50 \mathrm{~cd} / \mathrm{m}^{2}$, full field size $15^{\circ}$, and each check subtended $60^{\prime}$ and $15^{\prime}$. The mean luminance in the test room was kept at $80 \mathrm{~cd} / \mathrm{m}^{2}$, the contrast between the black and white squares was $85 \%$. The sweep speed, sensitivity and sweep duration and bandpass filters were $30 \mathrm{~ms} /$ division, 5 microV/ division, $300 \mathrm{~ms}$, and $2-100 \mathrm{~Hz}$, respectively. The subjects were asked to sit on a chair at a distance of $100 \mathrm{~cm}$ from the monitor, close one eye at a time while looking at a fixed point in the middle of the monitor, and relax. The rate of pattern reversal was $2 \mathrm{~Hz}$ and an average of two sets of 200 responses was recorded. Both eyes were separately tested.
Latencies (ms) of the major positive component (P100) and negative peaks (N75 and N135) and P100 amplitudes ( $\mu \mathrm{V}$ ) were determined. Abnormal P100 latencies were normally considered when the latency exceeding 2.5 to 3 SD beyond the mean, or beyond the $95^{\text {th }}$ to $99^{\text {th }}$ percentile. The laboratory values for P100 latency, determined previously in 28 healthy, 21-to 63-yearold controls, is $101.3 \pm 6.2 \mathrm{~ms}$ (mean \pm standard deviation), and therefore, the normal limit is $<116 \mathrm{~ms}$ (unpublished data). The VEP tests of the participants were evaluated by the same investigator who was blinded to the groups.

This study was approved by Yeditepe University Faculty of Medicine Local Ethics Committee (approval number: 2019920, date: 02.01.2019) and written informed consents were obtained from all participants.

\section{Statistical Analysis}

The sample size was determined by using the G*Power software within the inputs of $\alpha$ err prob: 0.05 , Power (1- $\beta$ err prob): 0.80 , and the effect size $d=0.8$ before the initiation of the study (11). SPSS 22.0 program was used for the analysis. The distribution of the variables was evaluated using the coefficient of variation, skewness-kurtosis, histogram and the Shapiro-Wilk normality test. Independent Samples t-test was used to compare the VEP values (P100, N75 and N135 waves, and amplitude of P100) of two groups (the right eye values of the patients were compared with the right eye values of the control group and the left eye values were compared with the left eye of the other group). The correlation between the P100 latencies and thyroid antibody levels in the HT group was evaluated using the Pearson correlation test. A p-value less than 0.05 was considered to be statistically significant.

\section{RESULTS}

Thirty HT patients and 30 control were included. Since all the patients were females, the control group was also composed of female volunteers. The reasons for and frequencies of the referral of the patients to the neurology clinic were as follows: Headache 18 (60\%), mild cognitive complaints (forgetfulness, decreased attention, and difficulty in concentration) $6(20 \%)$, sleep disturbances $3(10 \%)$, fatigue $2(6.7 \%)$ and dizziness $1(3.3 \%)$. There was no significant difference in age between the $\mathrm{HT}$ and control groups (38.93 \pm 8.73 and $33.80 \pm 8.77$ years, respectively). The mean duration of $\mathrm{HT}$ disease was $11.68 \pm 8.89$ years.

Mean serum TSH, FT4, TPOAb, and TgAB levels of HT patients were $2.7 \pm 1.88 \mathrm{ulU} / \mathrm{mL}, 1.16 \pm 0.21 \mathrm{ng} / \mathrm{dL}, 349.7 \pm 204.9 \mathrm{IU} / \mathrm{mL}$, and $192.09 \pm 168.16 \mathrm{IU} / \mathrm{mL}$, respectively. The visual acuities of all participants were 20/20.

Table 1 shows the comparisons of the mean latencies of P100, N75, and N135 waves, and P100 amplitudes of R and L eyes in the HT and control groups. There was no statistical difference between the latencies of the abovementioned waves and amplitudes between the two groups. In the HT group, P100 wave latencies were not significantly correlated with the levels of TPOAb $(r=0.08$, $p=0.71)$ and $\operatorname{TgAb}(r=-0.13, p=0.710)$. 


\begin{tabular}{|c|c|c|c|c|}
\hline VEP & SIDE & $\mathrm{HT}($ mean $\pm \mathrm{SD})$ & Control (mean \pm SD) & $p$ \\
\hline \multirow{2}{*}{ P100 latency (ms) } & $R$ & $108.13 \pm 4.3$ & $107.2 \pm 3.8$ & 0.412 \\
\hline & L & $108.2 \pm 4.4$ & $107.7 \pm 4.36$ & 0.662 \\
\hline \multirow{2}{*}{ N75 latency (ms) } & $R$ & $79.23 \pm 6.03$ & $79.91 \pm 5.50$ & 0.620 \\
\hline & L & $80.20 \pm 5.78$ & $78.90 \pm 6.17$ & 0.438 \\
\hline \multirow{2}{*}{ N135 latency (ms) } & $R$ & $141.8 \pm 11.2$ & $140.30 \pm 8.41$ & 0.630 \\
\hline & L & $142.4 \pm 10.2$ & $141.91 \pm 9.72$ & 0.869 \\
\hline \multirow{2}{*}{ P100 amplitude $(\mu \mathrm{V})$} & $R$ & $6.71 \pm 4.16$ & $8.20 \pm 3.32$ & 0.579 \\
\hline & L & $6.6 \pm 3.85$ & $6.92 \pm 2.90$ & 0.709 \\
\hline
\end{tabular}

VEP: visual evoked potentials, R: right eye, L: left eye, SD: standard deviation, ms: miliseconds, $\mu \mathrm{V}$ : microvolt, Independent Samples t-test, HT: Hashimoto's thyroiditis

\section{DISCUSSION}

Autoimmunity targeting the thyroid gland can also influence the CNS, and this association has been demonstrated by immunohistochemistry and imaging techniques (12-15). Animal models have shown that immune system activation can trigger inflammation in the CNS and influence animal behaviors (16). The effects of immune dysregulation on the CNS can be explained by the altered neural pathway functions and the blood-brain barrier damage as a result of the changes in various cytokines $(17,18)$. Positive glucocorticoid response and histologic findings of perivascular lymphocytic infiltration in $\mathrm{HE}$, the more overt CNS involvement of HT, may support the hypothesis based on the immunological explanation (19). Furthermore, in vitro studies showed that various antibodies, such as anti-ganglioside, antineural antibodies, and particularly TPOAbs, bind to CNS cells and impair myelin sheath damaging myelinogenesis $(6,20)$. The increased production of monocyte- and T-lymphocytederived cytokines in HT patients can also negatively affect some neurotransmitters playing role in various neuronal pathways (6).

In recent years, an increasing number of studies have reported insidious brain function abnormalities, only detected by specific tests, in euthyroid patients (6,21-23). Immunological mechanisms are the most probable reasons for these abnormalities, as shown by the studies indicating that TPOAb levels are higher in the patients with cognitive deficits than in the other HT patients without abnormalities $(6,22,23)$.

$H T$, the most common autoimmune thyroid disease, may also coexist with other autoimmune conditions $(24,25)$. In the context of this study, it is important that HT and autoimmune diseases of the CNS affecting the optic nerve and visual pathways, such as MS, are remarkably seen together. Since HE has several features resembling MS, such as common genetic loci, certain deregulated anti-inflammatory responses, elevated CSF oligoclonal bands, and demyelinating lesions on imaging studies, it is included in the differential diagnosis of MS, and both diseases have been suggested to have common similar pathological pathways in CNS involvement $(2,5,19,24,26)$.
The involvement of the optic nerve and visual pathways in autoimmune CNS disorders can be seen sub-clinically, as in MS, and is only demonstrated by special methods such as $\operatorname{VEP}(7,8)$. The hypothesis of our study was based on this information. Moreover, it has been shown that patients with recurrent and bilateral optic neuritis have a greater frequency of HT than other optic neuritis patients, despite normal magnetic resonance imaging (MRI) findings (27). In many cases, optic neuropathy in autoimmune thyroid diseases relates not only to thyroid hormones, but also to increased autoantibodies and can be seen in euthyroid patients (28).

VEP parameters, particularly latencies, may be influenced by hypothyroidism and hyperthyroidism. Although this situation is well documented in hypothyroidism, controversial results have been reported in hyperthyroidism (29-31). The prolongation of VEP latencies is the most common change, and it usually returns to the normal after the euthyroid state is achieved with the treatment in hypothyroid patients (32-34). Thyroid ophthalmopathies and exophthalmos are the main reasons of the VEP changes in hyperthyroid patients $(29,33,35)$. While the exact mechanisms remained elusive, compressive optic neuropathy, axoplasmic stasis, ischemia and mechanical stretch due to proptosis, and perineural inflammation have been proposed $(33,36)$. Decompressive surgical approaches and steroid treatment can greatly improve the VEP changes in hyperthyroid patients with ophthalmopathy $(37,38)$.

Our goal was to explore the presence of VEP changes as an indicator of the subclinical effects of HT on optic nerve and visual pathways. The pattern reversal VEP consists of a prominent positive component at approximately $100 \mathrm{~ms}$ (P100), followed by negative components (N75 and N135). Dysfunctions of the optic nerve, chiasma, and retrochiasmal pathways can be assessed using the VEP. Although a delayed P100 component often occurs in association with optic nerve diseases, it should not be considered pathognomonic for optic nerve diseases (29). VEP can be used to detect subclinical optic nerve demyelination. Even after the use of MRI increased, the VEP preserved its value in the diagnosis of demyelination because it has been shown that the specificity 
of the changes on MRI may be less than originally anticipated in many patients (39).

\section{Study Limitations}

We could not find any statistically significant difference between the groups in terms of the latencies of VEP waves and P100 amplitudes. These results may be due to the fact that the optic nerve and visual pathways are not significantly affected in euthyroid HT patients or the sensitivity of VEP is not sufficient $(8,40)$. There are some limitations of this study. The small number of patients and the lack of male euthyroid HT patients are some of our limitations.

\section{CONCLUSION}

The results of this study cannot support the hypothesis that VEP can be a screening test for the subclinical involvement of the optic nerve or visual pathways in euthyroid HT patients. As the possible reasons for these results, we suggested that there was no significant involvement in the aforementioned regions of CNS in euthyroid HT patients without visual symptoms and/or the lack of sufficient sensitivity of the VEP method in this regard.

Ethics Committee Approval: This study was approved by Yeditepe University Faculty of Medicine Local Ethics Committee (approval number: 2019-920, date: 02.01.2019)

Informed Consent: Written informed consents were obtained from all participants.

Peer-review: Externally peer-reviewed.

Author Contributions: Concept - H.Ş., B.E., Design - H.Ş., Supervision H.Ş., B.E., Materials - H.Ş., R.B., Data Collection and/or Processing - H.Ş., R.B., Analysis and/or Interpretation - H.Ş., B.E., Literature Search - H.Ş., Writing Manuscript - H.Ş., Critical Review - B.E.

Conflict of Interest: The authors have no conflict of interest to declare.

Financial Disclosure: The authors declared that this study has received no financial support.

\section{REFERENCES}

1. Nandi-Munshi D, Taplin CE. Thyroid-related neurological disorders and complications in children. Pediatr Neurol 2015; 52: 373-82.

2. Scolding $\mathrm{N}$. The differential diagnosis of multiple sclerosis. J Neurol Neurosurg Psychiatry 2001; 71(Suppl 2): 9-15.

3. Drakulic S, Toncev G, Vrndic O, Zivancevic Simonovic S. Neurological signs and symptoms in patients with autoimmune thyroid disease. Serbian J Exp Clin Res 2011; 12: 123-6.

4. Zarković M. Autoimmune thyroid disease and brain. Srp Arh Celok Lek 2005; 133(Suppl 1): 88-91.

5. Haralur Y, Mechtler LL. Neuroimaging of multiple sclerosis mimics. Neurol Clin 2020; 38: 149-70.

6. Leyhe T, Müssig K. Cognitive and affective dysfunctions in autoimmune thyroiditis. Brain Behav Immun 2014; 41: 261-6.

7. Demir S, Duzgun E, Kafadar C, Togrol E, Senol M. G, Cakir A. Evoked potentials and other guiding factors of conversion from radiologically isolated syndrome to definite multiple sclerosis. Med Sci Disc 2017; 4: 44-50.

8. Walsh P, Kane N, Butler S. The clinical role of evoked potentials. J Neurol Neurosurg Psychiatry 2005; 76(Suppl 2): 16-22.

9. Jha MK, Thakur D, Limbu N, Badhu BP, Paudel BH. Visual evoked potentials in primary open angle glaucoma. J Neurodegener Dis 2017; 2017: 9540609.
10. Odom JV, Bach $M$, Brigell $M$, Holder GE, McCulloch DL, Mizota A, Tormene AP. ISCEV standard for clinical visual evoked potentials: (2016 update). Doc Ophthalmol 2016; 133: 1-9.

11. Gautam V, Paudel BH, Lamsal M, Agrawal K, Jha MK, Maharjan S, et al. Visual evoked potentials' responses in hypothyroidism and hyperthyroidism. Int J Res Med Sci 2019; 7: 1589-93.

12. Pilhatsch $M$, Schlagenhauf $F$, Silverman $D$, Berman $S$, London $E D$, Martinez D, Whybrow PC, et al. Antibodies in autoimmune thyroiditis affect glucose metabolism of anterior cingulate. Brain Behav Immun 2014; 37: 73-7.

13. Cai YJ, Wang F, Chen ZX, Li L, Fan H, Wu ZB, et al. Hashimoto's thyroiditis induces neuroinflammation and emotional alterations in euthyroid mice. J Neuroinflammation 2018; 15: 299.

14. Piga M, Serra A, Deiana L, Loi GL, Satta L, Di Liberto $M$, et al. Brain perfusion abnormalities in patients with euthyroid autoimmune thyroiditis. Eur J Nucl Med Mol Imaging 2004; 31: 1639-44.

15. Quinque EM, Karger S, Arélin K, Schroeter ML, Kratzsch J, Villringer A. A Structural and functional MRI study of the brain. Cognition and mood in long-term adequately treated Hashimoto's thyroiditis. Psychoneuroendocrinology 2014; 42: 188-98.

16. Engler $H$, Doenlen $R$, Engler $A$, Riether $C$, Prager $G$, Niemi MB, et al. Acute amygdaloid response to systemic inflammation. Brain Behav Immun 2011; 25: 1384-92.

17. Wen J, Stock AD, Chalmers SA, Putterman $C$. The role of $B$ cells and autoantibodies in neuropsychiatric lupus. Autoimmun Rev 2016; 15: 8905.

18. Platt MP, Agalliu D, Cutforth T. Hello from the other side: how autoantibodies circumvent the blood-brain barrier in autoimmune encephalitis. Front Immunol 2017; 8: 442.

19. Tang Y, Xing Y, Lin MT, Zhang J, Jia J. Hashimoto's encephalopathy cases: Chinese experience. BMC Neurol 2012; 24: 12:60

20. Müssig K, Leyhe T, Holzmüller $S$, Klein R, Weinert $C$, Saur $R$, et al. Increased prevalence of antibodies to central nervous system tissue and gangliosides in Hashimoto's thyroiditis compared to other thyroid illnesses. Psychoneuroendocrinology 2009; 34: 1252-6.

21. Mazzù I, Mosti S, Caltagirone C, Carlesimo GA. Hashimoto's encephalopathy: Neuropsychological findings. Neurol Sci 2012; 33: 6536.

22. Leyhe $T$, Müssig K, Weinert $C$, Laske $C$, Häring HU, Saur R, et al. Increased occurrence of weaknesses in attention testing in patients with Hashimoto's thyroiditis compared to patients with thyroid illnesses. Psychoneuroendocrinology 2008; 33: 1432-6.

23. Müssig K, Künle A, Säuberlich AL, Weinert $C$, Ethofer $T$, Saur $R$, et al. Thyroid peroxidase antibody positivity is associated with symptomatic distress in patients with Hashimoto's thyroiditis. Brain Behav Immun 2012; 26: 559-63.

24. Perga S, Martire S, Montarolo F, Giordani I, Spadaro M, Bono G, et al. The footprints of poly-autoimmunity: evidence for common biological factors involved in multiple sclerosis and hashimoto's thyroiditis. Front Immunol 2018; 20: 9:311.

25. Nandi-Munshi D, Taplin CE. Thyroid-related neurological disorders and complications in children. Pediatr Neurol 2015; 52: 373-82.

26. Yamasaki R, Kira JI. Multiple Sclerosis. Adv Exp Med Biol 2019; 1190: $217-$ 47.

27. Kurne A, Karabudak R, Yalcin Cakmakli G, Gursoy Ozdemir Y, Aydin P, Ilksen Colpak $A$, et al. Recurrent optic neuritis: clues from a long-term follow up study of recurrent and bilateral optic neuritis patients. Eye Brain 2010; 5: 15-20.

28. Toyama S, Wakakura M, Chuenkongkaew W. Optic neuropathy associated with thyroid-related auto-antibodies. Neuro-Ophthalmology 2009; 25: 127-34.

29. Salvi M, Spaggiari E, Neri F, Macaluso C, Gardini E, Ferrozzi F, et al. The study of visual evoked potentials in patients with thyroid-associated ophthalmopathy identifies asymptomatic optic nerve involvement. J Clin Endocrinol Metab 1997; 82: 1027-30.

30. Nazliel B, Akbay E, Irkeç C, Yetkin I, Ersoy R, Törüner F. Pattern visual evoked potential (PVEP) evaluation in hypothyroidism. J Endocrinol Invest 2002; 25: 955-8. 
orbitopathy and subclinical optic nerve involvement. Doc Ophthalmol 2012; 125: 11-9.

37. Lipski A, Eckstein A, Esser J, Loesch C, Mann K, Mohr C, et al. Course of pattern-reversed visual evoked cortical potentials in 30 eyes after bony orbital decompression in dysthyroid optic neuropathy. $\mathrm{Br} \mathrm{J}$ Ophthalmol 2011; 95: 222-6.

38. Szanyi J, Kremlacek J, Kubova Z, Langrova J, Kuba M. A pilot study to monitor Graves' ophthalmopathy with a combination of pattern-reversal and motion-onset visual evoked potentials. J Clin Apher 2012; 27: 295301.

39. Holder GE. Electrophysiological assessment of optic nerve disease. Eye (Lond) 2004; 18: 1133-43.

40. American Clinical Neurophysiology Society. Guideline 9B: guidelines on visual evoked potentials. Am J Electroneurodiagnostic Technol 2006; 46: 254-74. 